\title{
Weighted Average Operators Generated by n-dimensional Overlaps and an Application in Decision Making
}

\author{
Ivanosca A. da Silva ${ }^{1}$ Benjamín Bedregal ${ }^{2}$ Humberto Bustince ${ }^{3}$ \\ ${ }^{1}$ Instituto Metrópole Digital, Universidade Federal do Rio Grande do Norte \\ ${ }^{2}$ Departamento de Informática e Matemática Aplicada, Universidade Federal do Rio Grande do Norte \\ ${ }^{3}$ Departamento de Automática y Computación, Universidad Pública de Navarra
}

\begin{abstract}
In this paper we provide a way to generate a class of weighted average operator from n-dimensional overlap functions and aggregation functions. These weighted average operators are used in an algorithm of a multiattribute group decision making problem based on decision matrix. An illustrative example is considered with the application of our method for two specific weighted average operators of this class. This result is compared with the ranking of other methods.
\end{abstract}

Keywords: n-dimensional overlaps, weighted average, multi-attribute group decision making

\section{Introduction}

Bustince et al. in [4] introduced a new class of aggregation functions called overlap functions, which are basically continuous, positive and commutative 2dimensional aggregation operators [3]. From them several theoretical and applied research on this kind of function have been made (see for example $[2,6,10,11,12$, $17,19])$. Overlap function has been applied in classification problems where the classes are not clearly separated (see for example [16]) and in decision making based on fuzzy preference relations (see for example [6]).

In [16], Gómez et al. extend the notion of overlap function for n-dimensional overlaps in order to measure the degree of overlapping of several classes in classification systems. They also study properties of migrativity, homogeneity and Lipschitz continuity for $\mathrm{n}$-dimensional overlaps. In addition it was also given an example in classification problems using Fuzzy Rule-Based Classification Systems where the use of n-dimensional overlap functions provide a better result than the product t-norm, which is the usual in this kind of problems.

On the other hand, Multi-attribute Group Decision Making consists in a choice of one or more alternatives among several ones by a group of decision makers (experts) who provide a matrix to express how much each alternative satisfies each one of the attributes considered $[5,15,14,22,23]$. Fuzzy logic, by its nature, has played an important role in the field of decision making, since it is usual that decision makers are subject to some uncertainty, which can be expressed in terms of fuzzy degrees.
This paper proposes a generalization of the weighted average operator, by considering a n-dimensional overlap function instead of the summatory. It is proved that the resulting operator results also in an aggregation operator and we apply one of this overlap generating weighted operator in an algorithm of multi-attribute group decision making based on decision matrices. The paper is organized as follows: In section 3 we consider the notion of n-dimensional overlap functions and a way to obtain n-dimensional overlap functions from overlap functions. Section 3 presents how to obtain an average operator from arbitrary overlaps and an algorithm to apply this operator in Multi-attribute Group Decision Making based on decision matrix. Finally section 4 presents the final considerations and future works.

\section{2. n-Dimensional Overlap Functions}

Overlap functions are special kinds of non-necessarily associative aggregation operators proposed in [4] in order to be applied in classification problems involving the overlap problem and when the associativity property is not strongly required, as in image processing and decision making based on aggregation operators [13].

Definition 2.1 A bivariate function $O:[0,1]^{2} \rightarrow[0,1]$ is said to be an overlap function if it satisfies the following conditions:

(01) O is symmetric;

(O2) $O(x, y)=0$ if and only if $x y=0$;

(O3) $O(x, y)=1$ if and only if $x y=1$;

(O4) O is non-decreasing;

(O5) $O$ is continuous.

Examples of overlap functions are the continuous t-norms with no zero divisors (property (O2)) and $O_{p}(x, y)=x^{p} y^{p}$, with $0<p$, which is not a t-norm when $p \neq 1$ [2].

It is well known that associative overlaps are positive continuous t-norms $[4,10]$. On the other hand, the associativity property of the t-norms allows to extend each $\mathrm{t}$-norm in a unique way to a n-dimensional operation as follows [18, Remark 1.10.(i)]: 


$$
T\left(x_{1}, \ldots, x_{n}\right)=T\left(x_{1}, T\left(x_{2}, \ldots, T\left(x_{n-1}, x_{n}\right) \cdots\right)\right.
$$

The unicity referred by Klement, Mesiar and Pap in [18], is in the sense that $T\left(x_{1}, T\left(x_{2}, \ldots, T\left(x_{n-1}, x_{n}\right) \cdots\right) \quad=\right.$ $T\left(T\left(\ldots T\left(T\left(x_{1}, x_{2}\right), \ldots, x_{n-1}\right), x_{n}\right) . \quad\right.$ However, for the case of overlaps which can not be associative, this is not possible. In particular, considering $O_{p}$ for $p=2$ and $n=3, O_{2}\left(0.4, O_{2}(0.5,0.8)\right)=0.16^{3} \neq$ $0.16^{2} * 0.25=\mathrm{O}_{2}\left(\mathrm{O}_{2}(0.4,0.5), 0.8\right)$.

On the other hand, Gómez et al. in [16], extended the notion of overlaps for $n$-dimensional overlaps as seen in the following definition:

Definition 2.2 A function $O:[0,1]^{n} \rightarrow[0,1]$ is said to be a $n$-dimensional overlap function if it satisfies the following conditions:

(01) O is symmetric;

(O2) $O\left(x_{1}, \ldots, x_{n}\right)=0$ if and only if $\prod_{i=1}^{n} x_{i}=0$;

(O3) $O\left(x_{1}, \ldots, x_{n}\right)=1$ if and only if $\prod_{i=1}^{n} x_{i}=1$;

(04) O is non-decreasing;

(O5) O is continuous.

A $n$-dimensional overlap is said strict if also satisfies the property

(06) $O\left(x_{1}, x_{2}, \ldots, x_{n}\right)<O\left(y, x_{2}, \ldots, x_{n}\right)$ when $\prod_{i=1}^{n} x_{i}>0 ;$ and $x_{1}<y$.

Let $O$ be an overlap and $n$ a positive natural number. Define the function, $O^{n}:[0,1]^{n} \rightarrow[0,1]$ given by

$O^{n}\left(x_{1}, \ldots, x_{n}\right)=O\left(x_{1}, O\left(x_{2}, \ldots, O\left(x_{n-1}, x_{n}\right) \cdots\right)\right.$

Observe that $O^{n}$ is not an overlap when $n \geq 3$ because the symmetry fails. The next theorem intends to recover the symmetry for $O^{n}$.

Theorem 2.1 Let $O$ be an overlap, $n$ a positive natural number and $A:[0,1]^{n !} \rightarrow[0,1]$ be a continuous aggregation function such that

(A1) if $A\left(x_{1}, \ldots, x_{n !}\right)=1$ then $x_{i}=1$ for some $i=$ $1, \ldots, n$ !

(A2) if $A\left(x_{1}, \ldots, x_{n !}\right)=0$ then $x_{i}=0$ for some $i=$ $1, \ldots, n !$.

Then the function $O_{A}^{n}:[0,1]^{n} \rightarrow[0,1]$ defined by

$$
O_{A}^{n}(\vec{x})=A\left(O^{n}\left(\vec{x}^{(1)}\right), \ldots, O^{n}\left(\vec{x}^{(n !)}\right)\right)
$$

is a $n$-dimensional overlap function when $\vec{x}^{(i)}$ is the $i$-th permutation $^{1}$ of $\vec{x} \in[0,1]^{n}$

\footnotetext{
${ }^{1}$ for any $n$-tuple of values there are just $n$ ! possible permutations.
}

Proof: Straightforward.

Notice that when $O$ is associative, i.e. is a t-norm, and $A$ is idempotent, i.e. is an average aggregation operator, then $O_{A}^{n}=O^{n}$. In fact, in general, it is desirable that $A$ is an average aggregation function.

Example 2.1 By considering $\mathrm{O}_{2}$ and the maximum aggregation operator $M$ we obtain the average operator

$$
O_{2 M}^{n}\left(x_{1}, \ldots, x_{n}\right)=O_{2}^{n}\left(x_{(1)}, \ldots, x_{(n)}\right)=\prod_{i=1}^{n} x_{(i)}^{2 i}
$$

where $\left(x_{(1)}, \ldots, x_{(n)}\right)$ is the permutation of $\left(x_{1}, \ldots, x_{n}\right)$ such that $x_{(i)} \geq x_{(i+1)}$ for each $i=1, \ldots, n-1$.

On the other hand,

$$
O_{0.5 M}^{n}\left(x_{1}, \ldots, x_{n}\right)=O_{0.5}^{n}\left(x_{(1)}, \ldots, x_{(n)}\right)=\prod_{i=1}^{n} x_{(i)}^{\frac{1}{2 i}}
$$

where $\left(x_{(1)}, \ldots, x_{(n)}\right)$ is the permutation of $\left(x_{1}, \ldots, x_{n}\right)$ such that $x_{(i)} \leq x_{(i+1)}$ for each $i=1, \ldots, n-1$.

Notice that there are n-dimensional overlaps which are not generated this way. For example, the Einstein product aggregation operator defined in [16] by

$$
\operatorname{EP}\left(x_{1}, \ldots, x_{n}\right)=\frac{\prod_{i=1}^{n} x_{i}}{1+\prod_{i=1}^{n}\left(1-x_{i}\right)}
$$

is a n-dimensional overlap which cannot be generated from an overlap and an aggregation operator according to Eq. (2).

\section{Application of n-dimensional overlaps in multiple attribute group decision making}

The weighted average (WA) and some variants of it are the most applied aggregation operators found in the literature. For example, they have been used in a wide range of different subjects such as statistics, economics and engineering [20]. In particular, there are several methods in decision making which consider a fuzzy or an extension of fuzzy framework that uses aggregation operators like the weighted average and ordered weighted average. For example, [7, 8] present a method which uses the generalization of the weighted average operator proposed by their respectives authors based on intervalvalued intuitionistic fuzzy values [1].

\subsection{Weighted average operators based on n-dimensional overlaps}

Let $F$ be a n-dimensional function satisfying $(\mathrm{O} 2)$ and $w=\left(w_{1}, \ldots, w_{n}\right)^{T}$ be a positive weighted vector, i.e. $w_{i} \neq 0$ for each $i=1, \ldots, n$ and $\sum_{i=1}^{n} w_{i}=1$. The weighted average operator based on $F$ and the positive 
weighted vector $w$ is the mapping $W A_{w}^{F}:[0,1]^{n} \rightarrow$ $[0,1]$ defined by

$$
W A_{w}^{F}\left(x_{1}, \ldots, x_{n}\right)=\frac{F\left(w_{1} x_{1}, \ldots, w_{n} x_{n}\right)}{F\left(w_{1}, \ldots, w_{n}\right)}
$$

Notice that if $F$ is the Łukasiewicz extended tconorm, i.e. $F\left(x_{1}, \ldots, x_{n}\right)=\min \left\{\sum_{i=1}^{n} x_{i}, 1\right\}$, then $W A_{w}^{F}$ is the usual weighted average operator for positive weighted vectors.

In the following we will consider the case when $F$ is a n-dimensional overlap.

Theorem 3.1 Let $O$ be a n-dimensional overlap and $w=\left(w_{1}, \ldots, w_{n}\right)^{T}$ be a positive weighted vector. Then $W A_{w}^{O}$ is a continuous $n$-ary aggregation function satisfying the property (O2). In addition, if $O$ satisfies (O6) then $W A_{w}^{O}$ satisfies (O3).

Proof: Since the product and $O^{n}$ are increasing, it is clear that $W A_{w}^{O}$ is also increasing. Moreover, $W A_{w}^{O}(0, \ldots, 0)=\frac{O(0, \ldots, 0)}{O\left(w_{1}, \ldots, w_{n}\right)}=\frac{0}{O\left(w_{1}, \ldots, w_{n}\right)}=0$ and $W A_{w}^{O}(1, \ldots, 1)=\frac{O\left(w_{1}, \ldots, w_{n}\right)}{O\left(w_{1}, \ldots, w_{n}\right)}=1$. Notice that $O\left(w_{1}, \ldots, w_{n}\right) \neq 0$ because each $w_{i} \neq 0$. Therefore, $W A_{w}^{O}$ is a continuous $n$-ary aggregation operator satisfying $(\mathrm{O} 2)$. Moreover, suppose that $O$ satisfies (O6). If $W A_{w}^{O}\left(x_{1}, \ldots, x_{n}\right)=1$ then, by (O2), we have that $x_{i} \neq 0$ for each $i=1, \ldots, n$ and by Eq. (3), $\frac{O\left(w_{1} x_{1}, \ldots, w_{n} x_{n}\right)}{O\left(w_{1}, \ldots, w_{n}\right)}=1$ and therefore $O\left(w_{1} x_{1}, \ldots, w_{n} x_{n}\right)=O\left(w_{1}, \ldots, w_{n}\right)$. Since $w_{i} x_{i} \leq$ $w_{i}$ for each $i=1, \ldots, n$, if for some $i=1, \ldots, n$, $x_{i} \neq 1$ then it is in contradiction with (O6). So, $W A_{w}^{O}\left(x_{1}, \ldots, x_{n}\right)=1$ iff $x_{i}=1$ for each $i=$ $1, \ldots, n$, i.e. $W A_{w}^{O}$ satisfies (O3).

Corollary 3.1 Let $O$ be a n-dimensional overlap and $w=\left(\frac{1}{n}, \ldots, \frac{1}{n}\right)^{T}$. If $O$ satisfies (O6) then $W A_{w}^{O}$ is a n-dimensional overlap.

Proof: Straightforward from Theorem $3.1 W A_{w}^{O}$ satisfies $(\mathrm{O} 2),(\mathrm{O} 3),(\mathrm{O} 4)$ and $(\mathrm{O} 5)$. The symmetry, i.e. $(\mathrm{O} 1)$, follows from the symmetry of $O$ and the fact that, in this case, the $w_{i}^{\prime} s$ of Eq. (3) are all equals.

\subsection{Multiple attribute group decision making based on $W A_{w}^{O}$}

A solution for a multiple attribute group decision making problem (MAGDMP) is a method to choose a "good" alternative from a set of them, considering a set of attributes for the alternatives and the opinion of a group of experts. Formally, in a MAGDMP we have a finite set $X=\left\{x_{1}, \ldots, x_{n}\right\}$ of feasible alternatives, a set $A=\left\{a_{1}, \ldots, a_{m}\right\}$ of attributes with their associated positive weighted vector $w=\left(w_{1}, \ldots, w_{m}\right)^{T}$, and a set $d=\left\{d_{1}, \ldots, d_{t}\right\}$ of decision makers and a positive weighted vector $\omega=\left(\omega_{1}, \ldots, \omega_{t}\right)^{T}$ of decision makers satisfying the usual condition $\sum_{i=1}^{k} \omega_{i}=1$. The method must choose the alternative which "better" satisfies all the attributes. For that, each decision maker $d_{k}$ determines a decision matrix $M^{(k)}=\left(m_{i j}^{(k)}\right)_{n \times m}$, where the rows represent the alternatives and the columns represent the attributes. In classic decision making, according to the opinion of the decision makers $d_{k}$, the position $m_{i j}^{(k)}$ of $M^{(k)}$ has the value 1 , in case the alternative $x_{i}$ has the attribute $a_{j}$ and zero otherwise. Nevertheless, in several situations, some attributes are inherently fuzzy, for example "quality of construction project", and so it must be dealt as a fuzzy set. In this case, the value in the position $m_{i j}^{(k)}$ would be the membership degree, i.e. a value in $[0,1]$, of the alternative $x_{i}$ to the fuzzy set associated to the attribute $a_{j}$. In general, we have two types of attributes: benefit and cost. For example, the attribute "quality of construction project" is a benefit attribute whereas "risk of investment" is a cost attribute. Let $I$ be the set of index of the benefit attributes.

We propose the following solution for MAGDMP:

Step 1 Transform each decision matrix $M^{(k)}$ into the standard decision matrix $R^{(k)}=\left(r_{i j}^{(k)}\right)_{n \times m}$ as follows:

$$
r_{i j}^{(k)}= \begin{cases}m_{i j}^{(k)} & \text { if } j \in I \\ 1-m_{i j}^{(k)} & \text { if } j \notin I\end{cases}
$$

Step 2 Given an n-dimensional overlap $O$, in order to obtain a collective decision matrix $C=\left(c_{i j}\right)_{n \times m}$, aggregate the standard decision matrices with $W A_{\omega}^{O}$ as follows:

$$
c_{i j}=W A_{\omega}^{O}\left(r_{i j}^{(1)}, \ldots, r_{i j}^{(t)}\right)
$$

Step 3 For each alternative aggregate the membership degrees to each attribute by using $W A_{w}^{O}$, i.e. for each alternative $x_{i}$ determine the collective overall preference value cop $_{i}$

$$
\operatorname{cop}_{i}=W A_{w}^{O}\left(c_{i 1}, \ldots, c_{i m}\right)
$$

Step 4 Rank the alternatives in decreasing order with respect to the collective overall preference values and choose the alternative with greatest value.

\subsection{Illustrative example}

We will consider the illustrative example used in $[9,21]$ to show how to use our method.

Suppose that an investor intends to invest part of his capital in a company. By a market analysis the investor reduces the spectrum of possible companies into six:

1. A chemical company, denoted by $x_{1}$.

2. A food company denoted by $x_{2}$.

3. A computer company denoted by $x_{3}$.

4. A car company denoted by $x_{4}$.

5. A furniture company denoted by $x_{5}$.

6. A pharmaceutical company denoted by $x_{6}$. 
Table 1: Assessment of expert $e_{1}$.

\begin{tabular}{|c|cccccc|}
\hline$M^{(1)}$ & $a_{1}$ & $a_{2}$ & $a_{3}$ & $a_{4}$ & $a_{5}$ & $a_{6}$ \\
\hline$x_{1}$ & 0.7 & 0.8 & 0.6 & 0.7 & 0.5 & 0.9 \\
$x_{2}$ & 0.8 & 0.6 & 0.9 & 0.7 & 0.6 & 0.7 \\
$x_{3}$ & 0.5 & 0.4 & 0.8 & 0.3 & 0.8 & 0.8 \\
$x_{4}$ & 0.6 & 0.7 & 0.6 & 0.7 & 0.8 & 0.6 \\
$x_{5}$ & 0.9 & 0.8 & 0.4 & 0.7 & 0.7 & 0.8 \\
$x_{6}$ & 0.8 & 0.3 & 0.7 & 0.7 & 0.6 & 0.7 \\
\hline
\end{tabular}

Table 2: Assessment of expert $e_{2}$.

\begin{tabular}{|c|cccccc|}
\hline$M^{(2)}$ & $a_{1}$ & $a_{2}$ & $a_{3}$ & $a_{4}$ & $a_{5}$ & $a_{6}$ \\
\hline$x_{1}$ & 0.6 & 0.8 & 0.5 & 0.6 & 0.4 & 0.8 \\
$x_{2}$ & 0.7 & 0.6 & 0.8 & 0.6 & 0.7 & 0.7 \\
$x_{3}$ & 0.7 & 0.6 & 0.8 & 0.7 & 0.8 & 0.8 \\
$x_{4}$ & 0.6 & 0.7 & 0.5 & 0.6 & 0.8 & 0.7 \\
$x_{5}$ & 0.7 & 0.8 & 0.7 & 0.7 & 0.6 & 0.8 \\
$x_{6}$ & 0.6 & 0.4 & 0.8 & 0.7 & 0.6 & 0.7 \\
\hline
\end{tabular}

The investor is helped by a group of three experts or decision makers $\left(e_{1}, e_{2}\right.$ and $\left.e_{3}\right)$ with the following weights $\omega=(0.3,0.3,0.4)$. The group of experts establish that six attributes will be used to evaluate the investments.

The benefit attributes are:

$\left.a_{1}\right)$ Benefits in the short term.

$a_{2}$ ) Benefits in the middle term.

$a_{3}$ ) Benefits in the long term.

The cost attributes are:

$\left.a_{4}\right)$ Risk of the investment.

$a_{5}$ ) Difficulty of the investment.

$a_{6}$ ) Other unfavorable factors on the investment.

Tables 1, 2 and 3 describes the assesses of the experts of how much the investment satisfies each attribute, i.e. they are the decision matrix of each expert.

We normalize these decision matrices resulting in the standard decision matrices $R^{(1)}, R^{(2)}$ and $R^{(3)}$ described, respectively, in Tables 4, 5 and 6 .

In the following, the collective decision matrix from the standard decision matrices is obtained by using $W A_{\omega}^{O_{0.5 M}^{3}}$ resulting in the matrix of Table 7 (with the values rounded in the third decimal digit).

The next step consists in determining the collective overall preference vector $C O P$ by considering $W A_{w}^{O_{0.5 M}^{5}}$ where $w=(0.1,0.1,0.2,0.2,0.2,0.2)$. The result is in Table 8.

Table 3: Assessment of expert $e_{3}$.

\begin{tabular}{|c|cccccc|}
\hline$M^{(3)}$ & $a_{1}$ & $a_{2}$ & $a_{3}$ & $a_{4}$ & $a_{5}$ & $a_{6}$ \\
\hline$x_{1}$ & 0.7 & 0.6 & 0.6 & 0.6 & 0.4 & 0.7 \\
$x_{2}$ & 0.7 & 0.6 & 0.7 & 0.6 & 0.6 & 0.7 \\
$x_{3}$ & 0.6 & 0.5 & 0.8 & 0.5 & 0.8 & 0.8 \\
$x_{4}$ & 0.6 & 0.7 & 0.7 & 0.5 & 0.8 & 0.6 \\
$x_{5}$ & 0.7 & 0.8 & 0.6 & 0.7 & 0.6 & 0.8 \\
$x_{6}$ & 0.4 & 0.5 & 0.9 & 0.7 & 0.6 & 0.6 \\
\hline
\end{tabular}

Table 4: Standardized decision matrix of expert $e_{1}$.

\begin{tabular}{|c|cccccc|}
\hline$R^{(1)}$ & $a_{1}$ & $a_{2}$ & $a_{3}$ & $a_{4}$ & $a_{5}$ & $a_{6}$ \\
\hline$x_{1}$ & 0.7 & 0.8 & 0.6 & 0.3 & 0.5 & 0.1 \\
$x_{2}$ & 0.8 & 0.6 & 0.9 & 0.3 & 0.4 & 0.3 \\
$x_{3}$ & 0.5 & 0.4 & 0.8 & 0.7 & 0.2 & 0.2 \\
$x_{4}$ & 0.6 & 0.7 & 0.6 & 0.3 & 0.2 & 0.4 \\
$x_{5}$ & 0.9 & 0.8 & 0.4 & 0.3 & 0.3 & 0.2 \\
$x_{6}$ & 0.8 & 0.3 & 0.7 & 0.3 & 0.4 & 0.3 \\
\hline
\end{tabular}

Table 5: Standardized decision matrix of expert $e_{2}$.

\begin{tabular}{|c|cccccc|}
\hline$R^{(2)}$ & $a_{1}$ & $a_{2}$ & $a_{3}$ & $a_{4}$ & $a_{5}$ & $a_{6}$ \\
\hline$x_{1}$ & 0.6 & 0.8 & 0.5 & 0.4 & 0.6 & 0.2 \\
$x_{2}$ & 0.7 & 0.6 & 0.8 & 0.4 & 0.3 & 0.3 \\
$x_{3}$ & 0.7 & 0.6 & 0.8 & 0.3 & 0.2 & 0.2 \\
$x_{4}$ & 0.6 & 0.7 & 0.5 & 0.4 & 0.2 & 0.3 \\
$x_{5}$ & 0.7 & 0.8 & 0.7 & 0.3 & 0.4 & 0.2 \\
$x_{6}$ & 0.6 & 0.4 & 0.8 & 0.3 & 0.4 & 0.3 \\
\hline
\end{tabular}

Table 6: Standardized decision matrix of expert $e_{3}$.

\begin{tabular}{|c|cccccc|}
\hline$R^{(3)}$ & $a_{1}$ & $a_{2}$ & $a_{3}$ & $a_{4}$ & $a_{5}$ & $a_{6}$ \\
\hline$x_{1}$ & 0.7 & 0.6 & 0.6 & 0.4 & 0.6 & 0.3 \\
$x_{2}$ & 0.7 & 0.6 & 0.7 & 0.4 & 0.4 & 0.3 \\
$x_{3}$ & 0.6 & 0.5 & 0.8 & 0.5 & 0.2 & 0.2 \\
$x_{4}$ & 0.6 & 0.7 & 0.7 & 0.5 & 0.2 & 0.4 \\
$x_{5}$ & 0.7 & 0.8 & 0.6 & 0.3 & 0.4 & 0.2 \\
$x_{6}$ & 0.4 & 0.5 & 0.9 & 0.3 & 0.4 & 0.4 \\
\hline
\end{tabular}

Table 7: Collective decision matrix

\begin{tabular}{|l|cccccc|}
\hline$C$ & $a_{1}$ & $a_{2}$ & $a_{3}$ & $a_{4}$ & $a_{5}$ & $a_{6}$ \\
\hline$x_{1}$ & 0.703 & 0.706 & 0.607 & 0.412 & 0.607 & 0.250 \\
$x_{2}$ & 0.746 & 0.626 & 0.785 & 0.412 & 0.412 & 0.331 \\
$x_{3}$ & 0.631 & 0.534 & 0.815 & 0.536 & 0.229 & 0.229 \\
$x_{4}$ & 0.626 & 0.721 & 0.656 & 0.460 & 0.229 & 0.416 \\
$x_{5}$ & 0.768 & 0.815 & 0.608 & 0.332 & 0.412 & 0.229 \\
$x_{6}$ & 0.614 & 0.460 & 0.845 & 0.332 & 0.432 & 0.383 \\
\hline
\end{tabular}

Table 8: Collective overall preference vector

\begin{tabular}{|c|cccccc|}
\hline$C O P$ & $x_{1}$ & $x_{2}$ & $x_{3}$ & $x_{4}$ & $x_{5}$ & $x_{6}$ \\
\hline $\operatorname{cop}_{i}$ & 0.658 & 0.674 & 0.594 & 0.621 & 0.694 & 0.566 \\
\hline
\end{tabular}


Table 9: Summary of the ranking obtained in [21], [9] and with the proposed method.

\begin{tabular}{|l|l|}
\hline Methods & \multicolumn{1}{|c|}{ Ranking } \\
\hline Maximum & $x_{2} \succ x_{4} \succ x_{5} \succ x_{1} \succ x_{3} \succ x_{6}$ \\
Minimum & $x_{3} \sim x_{5} \succ x_{1} \succ x_{4} \succ x_{2} \succ x_{6}$ \\
NHD & $x_{5} \succ x_{2} \succ x_{3} \succ x_{4} \succ x_{1} \succ x_{6}$ \\
WHD & $x_{5} \succ x_{3} \succ x_{2} \succ x_{4} \succ x_{6} \succ x_{1}$ \\
Step-IOWAD & $x_{5} \succ x_{4} \succ x_{6} \succ x_{2} \succ x_{3} \succ x_{1}$ \\
Hurwicz & $x_{3} \succ x_{2} \succ x_{6} \succ x_{4} \succ x_{1} \succ x_{5}$ \\
OWAD & $x_{5} \succ x_{3} \succ x_{2} \succ x_{4} \succ x_{1} \succ x_{6}$ \\
AOWAD & $x_{5} \succ x_{2} \succ x_{4} \succ x_{3} \succ x_{6} \succ x_{1}$ \\
IOWAD & $x_{5} \succ x_{3} \succ x_{2} \succ x_{1} \succ x_{4} \succ x_{6}$ \\
AIOWAD & $x_{5} \succ x_{2} \succ x_{6} \succ x_{4} \succ x_{3} \succ x_{1}$ \\
Median-IOWAD & $x_{5} \succ x_{1} \succ x_{6} \succ x_{2} \succ x_{4} \succ x_{3}$ \\
Olympic-IOWAD & $x_{5} \succ x_{4} \succ x_{1} \succ x_{2} \succ x_{3} \succ x_{6}$ \\
[9] & $x_{2} \sim x_{3} \succ x_{6} \succ x_{4} \succ x_{1} \succ x_{5}$ \\
$W A_{\omega}^{O_{0.5 M}^{3}}$ & $x_{5} \succ x_{2} \succ x_{1} \succ x_{4} \succ x_{3} \succ x_{6}$ \\
$W A_{\omega}^{O_{2 M}^{3}}$ & $x_{2} \succ x_{1} \succ x_{5} \succ x_{4} \succ x_{3} \succ x_{6}$ \\
\hline
\end{tabular}

Based on Table 8, we obtain the following rank of the alternatives:

$$
x_{5} \succ x_{2} \succ x_{1} \succ x_{4} \succ x_{3} \succ x_{6}
$$

If we use the aggregation operators $W A_{\omega}^{O_{2 M}^{3}}$ and $W A_{w}^{O_{2 M}^{5}}$ instead of $W A_{\omega}^{O_{2 M}^{3}}$ and $W A_{w}^{O_{2 M}^{5}}$, respectively, we obtain the following ranking:

$$
x_{2} \succ x_{1} \succ x_{5} \succ x_{4} \succ x_{3} \succ x_{6}
$$

Observe that this two ranking agree in the last three positions, but the alternatives $x_{5}$, the winner of the previous ranking, now is in the third position.

On the other hand, the Table 9 adds this two ranking and the ranking obtained in [9] to the Table 10 of [21] which contains the result of twelve methods for this same MAGDMP. Analysing this table, we can see that the fifteen methods return fifteen different rankings. Nevertheless, the most (ten) agrees that the best alternative is $x_{5}$ and eight that the worst alternative is $x_{6}$. So, the ranking obtained with $W A_{\omega}^{O_{0.5 M}^{3}}$ seem more reasonable than the obtained with $W A_{\omega}^{O_{2 M}^{3}}$.

\section{Final remarks}

We propose a method to generate average aggregation functions from n-dimensional overlaps and aggregation operators, and also propose a method for multi-attribute group decision making problems, based on the weighted average generated by the n-dimensional overlaps $O_{0.5}$ and $\mathrm{O}_{2}$ joint with the aggregation operator "Maximum". We also consider a multi-attribute group decision making problem which was studied in [21] resulting in 12 differents ranking. The ranking obtained here for $O_{0.5}$ agrees with the most of them in the best and in the worst alternative which is just an evidence that this method result in a reasonable ranking. The goal of introduce this new method is not be the best method for MAGDMP, neither give the best ranking for a particular example neither be better than other methods, but just contribute with a new tool to provide new evidences for the decision maker can select good alternatives. In fact, in general, there is no way to validate the quality of a ranking and therefore of a method for decision making problems, but when we have several rankings obtained by reasonable methods, we can determine a good ranking or a partial good ranking with the information provided for all the methods used.

As a future work we will compare the result with the weighted average generated by the n-dimensional overlap $\mathrm{O}_{0.5}$ and $\mathrm{O}_{2}$ and the maximum aggregation operator with the generated by other n-dimensional overlaps and aggregation functions. In addition, we will also study the generation of like OWA operators from ndimensional overlaps and apply them in multi-attribute group decision making and addapt the method for group decision making problems based on preference relations as in $[6,8,24]$.

\section{Acknowledgements}

This work was partially supported by the Brazilian funding agency $\mathrm{CNPq}$ - Conselho Nacional de Desenvolvimento Científico e Tecnológico), under Proc. No. 307681/2012-2 and No. 406503/2013-3.

\section{References}

[1] K. Atanassov and G. Gargov. Interval valued intuitionistic fuzzy sets. Fuzzy Sets and Systems, v. 31, n. 3, p. 343-349, 1989.

[2] B. Bedregal, G.P. Dimuro, H. Bustince and E. Barrenechea. New Results on overlaps and grouping functions. Information Sciences v. 249, p. 148 170, 2013.

[3] G. Beliakov, A. Pradera and T. Calvo. Aggregation Functions: A Guide for Practitioners, Springer, Berlin, 2007.

[4] H.Bustince, J.Fernandez, R.Mesiar, J.Montero and R.Orduna. Overlap functions. Nonlinear Analysis, v. 72, p. 1488-1499, 2010.

[5] H. Bustince, M. Galar, B. Bedregal, SA. Kolesárová and R. Mesiar. A New Approach to Interval-Valued Choquet Integrals and the Problem of Ordering in Interval-Valued Fuzzy Set Applications. IEEE Trans. on Fuzzy Systems, v. 21, n. 6 , p. 1150-1162, 2013.

[6] H. Bustince, M. Pagola, R. Mesiar, E. Hüllermeier and F. Herrera. Grouping, Overlap, and Generalized Bientropic Functions for Fuzzy Modeling of Pairwise Comparisons. IEEE T. Fuzzy Systems, v. 20, n. 3, p. 405-415, 2012.

[7] S.M. Chen, L.W. Lee, H.C. Liu and S.W. Yang. Multiattribute decision making based on intervalvalued intuitionistic fuzzy values. Expert Systems with Applications, v. 39, n. 12, p. 10343-10351, 2012.

[8] I.A. da Silva, B. Bedregal, R.H.N. Santiago and A.D. Dória Neto. A new method for intervalvalued intuitionistic group decision making. In: 
Segundo Congresso Brasileiro de Sistemas Fuzzy, 2012, Natal-RN. Recentes Avanços em Sistemas Fuzzy. São Carlos-SP: SBMAC, 2012. v. 1. p. 282294.

[9] I.A. da Silva, B.C. Bedregal, C.G. da Costa, E. Palmeira and M.P. da Rocha. Pseudo-Uninorms and Atanassov's Intuitionistic Pseudo-Uninorms. J. of Intelligent and Fuzzy Systems, 2015. doi: 10.3233/IFS-151593.

[10] G.P. Dimuro and B.C. Bedregal. Archimedean overlap functions: The ordinal sum and the cancellation, idempotency and limiting properties. Fuzzy Sets and Systems, v. 252, p. 39-54, 2014.

[11] G.P. Dimuro and B.C. Bedregal. On residual implications derived from overlap functions. Information Sciences, 2015. doi:10.1016/j.ins.2015.03.049

[12] G.P. Dimuro, B.C. Bedregal, H. Bustince, M.J. Asiáin and R. Mesiar. On additive generators of overlap functions. Fuzzy Sets and Systems, 2015. doi:10.1016/j.fss.2015.02.008.

[13] G.P. Dimuro, B. Bedregal and R.H.N. Santiago. On (G,N)-implications derived from grouping functions. Information Sciences, v. 219, p. 1-17, 2014.

[14] B. Feng and F. Lai. Multi-attribute group decision making with aspirations: A case study. Omega, v. 44, p. 136-147, 2014.

[15] J. Figueira, S. Greco and M. Ehrgott (eds.). Multiple Criteria Decision Analysis: State of the arts surveys. Springer, Berlin-Heildelberg, 2007.

[16] D. Gómez, J.T. Rodríguez, J. Montero, H. Bustince and E. Barrenechea. n-Dimensional overlap functions. Fuzzy Sets and Systems, 2015. doi:10.1016/j.fss.2014.11.023

[17] A. Jurio, H. Bustince, M. Pagola, A. Pradera and R.R. Yager. Some properties of overlap and grouping functions and their application to image thresholding. Fuzzy Sets and Systems, v. 229, p. 69-90, 2013.

[18] E.P. Klement, R. Mesiar and E. Pap. Triangular Norms. Kluwer Academic Publishers, Dordrecht, 2000.

[19] N. Madrid, A. Burusco, H. Bustince, J. Fernandez and I. Perfilieva. Upper bounding overlaps by groupings. Fuzzy Sets and Systems, v. 264, p. 7699, 2015.

[20] J.M. Merigó. A unified model between the weighted average and the induced OWA operator. Expert Systems with Applications, v. 38, n. 9, p. 11560-11572, 2011.

[21] J.M. Merigó and M. Casanova. Decision-Making with distance measures and induced aggregation operators. Computer \& Industrial Engineering, v. 60, p. 66-76, 2011.

[22] S.M. Mousavi, F. Jolai and R. TavakkoliMoghaddam. A Fuzzy Stochastic Multi-Attribute Group Decision-Making Approach for Selection Problems. Group Decision and Negotiation, v. 22, n. 2, p. 207-233, 2013.

[23] J. Pang and J. Liang. Evaluation of the results of multi-attribute group decision-making with lin- guistic information. Omega, v. 40, p. 294-301, 2012.

[24] D. Paternain, A. Jurio, E. Barrenechea, H. Bustince, B. Bedregal and E. Szmidt. An alternative to fuzzy methods in decision-making problems. Expert Systems with Applications, 39: 7729 7735, 2012. 